\title{
Seminare / Séminaires 2009
}

\section{Praxiseröffnung/-übernahme}

\section{Themen}

Juristische Aspekte (Praxisbewilligung, Zulassung zur Sozialversicherung, Vertragswesen), Gesellschaftsformen/Ehe- und Erbrecht (Trennung Privat- vom Geschäftsvermögen, Ehegüterstand, Erbschaftsplanung), Praxiseinrichtung (Inneneinrichtung, Kostenberechnung), Praxisadministration (Leistungserfassungsund Abrechnungssysteme, Praxismarketing), Unternehmensbewertung einer Arztpraxis (Berechnung und Beurteilung des Unternehmenswertes), Finanzierung der Arztpraxis (Businessplan, Kredite, Absicherungsmöglichkeiten), Versicherungen/Vorsorge/Vermögen (Personen- und Sachversicherungen, Vorsorgeplanung).

\section{Sponsoren}

Die Kosten werden durch diverse Sponsoren (siehe www.fmhservices.ch) gedeckt.

\section{Daten}

\begin{tabular}{llll} 
K04 & $\begin{array}{l}\text { Donnerstag, 3. September 2009 } \\
\text { Zürich }\end{array}$ & FMT \\
9.00-16.30 Uhr & \\
K05 & $\begin{array}{l}\text { Donnerstag, 5. November 2009 } \\
\text { Basel }\end{array}$ & $\begin{array}{l}\text { Hotel } \\
\text { 9.00-16.30 Uhr }\end{array}$ & Victoria \\
\hline
\end{tabular}

\section{Praxisübergabe}

\section{Hinweis}

Vor allem aus steuerplanerischer Sicht lohnt es sich, sich bereits frühzeitig (5-10 Jahre) mit diesem Thema auseinanderzusetzen.

\section{Themen}

Juristische Aspekte (Praxisübergabevertrag, allg. Vertragswesen, Übergabe der Krankengeschichten), Unternehmensbewertung einer Arztpraxis (Berechnung Inventarwert und Goodwill als Verhandlungsbasis), Versicherungen/Vorsorge/Vermögen (Übergabe/Auflösung von Versicherungsverträgen, Pensions- und Finanzplanung), Steuern (Steueraspekte bei der Praxisübergabe, Optimierung der steuerlichen Auswirkungen, Liquidations- und Grundstückgewinnsteuer, Bestimmung des optimalen Übergabezeitpunktes)

\section{Sponsoren}

Die Kosten werden durch diverse Sponsoren (siehe www.fmhservices.ch) gedeckt.

\section{Daten}

\begin{tabular}{|c|c|c|c|}
\hline К09 & Donnerstag & 10. September 2009 & FMT \\
\hline & Zürich & 13.30-18.00 Uhr & SAS Hotel \\
\hline K & Donnersta & 12. November 2009 & Hotel \\
\hline
\end{tabular}

\section{Finanz- und Steuerplanung}

\section{Themen}

Finanzplanung (Businessplan, buchhalterische Massnahmen vor Praxiseröffnung/-übernahme, Standardkontenplan für Ärzte, System der doppelten Buchhaltung, EDV-unterstützte Buchführungslösung), Steuern (Steueraspekte bei Eintritt in die Selbständigkeit, Steuerfallen und Steuerrisiken, optimierte Steuerplanung).

\section{Sponsoren}

Die Kosten werden durch diverse Sponsoren (siehe www.fmhservices.ch) gedeckt.

\section{Daten}

K12 Donnerstag, 17. September 2009 Schmiedstube
Bern $\quad 13.30-18.00 \mathrm{Uhr}$

\section{Praxiscomputerworkshop}

\section{Inhalt}

Die Workshopteilnehmer/innen erhalten im 1. Teil eine Einführung in die Anforderungen an ein Praxisinformationssystem. Anhand einer modernen vernetzten Praxisinfrastruktur werden die Beurteilungskriterien für eine praxis- und zukunftstaugliche Softwarelösung dargestellt. Checklisten sollen die schnelle Orientierung unterstützen und bei der Beurteilung und Wahl des Produkts konkrete Hilfe bieten. In Zusammenarbeit mit SGAM.Informatics werden die zentralen Elemente der elektronischen Krankengeschichte aufgezeigt. Ein Erfahrungsbericht eines EDV-Anwenders (Arzt) rundet den 1. Teil ab. Der 2. Teil umfasst die Präsentation von sechs Praxisadministrationssoftwarelösungen (Leistungserfassung, elektronisches Abrechnen unter Einbezug der TrustCenter, Agendaführung, Statistiken, Laborgeräteeinbindung, elektronische Krankengeschichte, Finanzbuchhaltungslösungen usw.).

\section{Ziel}

Die Teilnehmer/innen erhalten einen Anforderungskatalog, der ihnen erlaubt, ihre Vorstellungen für ein modernes Praxisinformationssystem besser zu formulieren und diese dem Softwarehersteller zu dessen Vor- 
bereitung zu kommunizieren. Zudem erhalten sie einen ersten Überblick über führende Softwarelösungen.

\section{Daten}

K15 Donnerstag, 26. November 2009 Stadttheater Olten 9.30-16.30 Uhr Olten

\section{Röntgen in der Arztpraxis}

\section{Teilnehmende}

Das Seminar richtet sich an Ärztinnen und Ärzte mit bestehender Praxis und an solche, die vor einer Praxiseröffnung oder Praxisübernahme stehen. Das Seminar ist auf maximal 15 Teilnehmer/innen beschränkt.

\section{Themen}

Vom konventionellen zum digitalen Röntgen, Rentabilität Röntgen in der Arztpraxis, neue Vorschriften seit 1. Januar 2008, Evaluation und Beschaffung neuer oder gebrauchter Anlagen, komplette Marktübersicht mit Preisen und Leistungskomponenten. Die Seminarteilnehmer erstellen und bearbeiten innerhalb des Seminars digitale Röntgenbilder und erhalten eine komplexe Dokumentation über alle Themen ein Vademekum rund ums Röntgen.

\section{Kosten}

Fr. 300.- (inkl. sämtlicher Kursunterlagen und Verpflegung).

\section{Daten}

K16 Donnerstag, 27. August 2009 WIROMA AG Niederscherli $9.30-16.00 \mathrm{Uhr}$

\section{Ouverture et reprise d'un cabinet médical}

\section{Contenu}

Business plan (préparation du plan de financement et crédit d'exploitation, financement par la banque). Aménagement (implantation, projet et concept d'aménagement, choix du mobilier, budget). Estimation d'un cabinet (inventaire et goodwill). Administration d'un cabinet médical (dans le cabinet, par la banque). Assurances (toutes les assurances à l'intérieur et autour du cabinet). Passage du statut de salarié à celui d'indépendant et fiscalité.

\section{Sponsors}

Les coûts sont pris en charge par divers sponsors (voir www.fmhservices.ch).

\begin{tabular}{|c|c|c|}
\hline \multicolumn{3}{|c|}{ Dates } \\
\hline K22 & Jeudi 3 septembre 2009 & Ramada \\
\hline & Genève $\quad 17.00-21.30 \mathrm{~h}$ & Park Hôtel \\
\hline \multirow[t]{2}{*}{$\mathrm{K} 23$} & Jeudi 19 novembre 2009 & Au Parc \\
\hline & Fribourg $\quad 17.00-21.30 \mathrm{~h}$ & Hôtel \\
\hline
\end{tabular}

\section{Remise d'un cabinet médical}

\section{Contenu}

Aspects juridiques (autour du contrat de remise/ reprise), Estimation d'inventaire et goodwill d'un cabinet, Assurances (prévoyance, assurances à l'intérieur et autour du cabinet), Conséquences fiscales d'une remise.

\section{Sponsors}

Les coûts sont pris en charge par divers sponsors (voir www.fmhservices.ch).

\section{Dates}

$\begin{array}{lll}\text { K25 Jeudi } 12 \text { novembre } 2009 & \text { Ramada } \\ \text { Genève } & 17.00-21.30 \mathrm{~h} & \text { Park Hôtel }\end{array}$

\section{Anmeldung und Auskunft / Inscription et information}

Internet unter www.fmhservices.ch oder FMH Consulting Services, Sandra Stadelmann, Burghöhe 1, 6208 Oberkirch, Tel. 04192500 77, Fax 0419210586

\section{Hinweis/Remarque}

Bei sämtlichen Seminaren, bei denen die Kosten teilweise oder gänzlich von Seminarsponsoren gedeckt werden, werden die Teilnehmeradressen den jeweiligen Sponsoren zur Verfügung gestellt.

Les adresses des participants aux séminaires dont les coûts sont couverts en partie ou totalement par des sponsors sont communiquées aux sponsors concernés.

\section{Annullierungsbedingungen /}

\section{Conditions d'annulation}

Bei Abmeldungen oder Fernbleiben werden folgende Unkostenbeiträge erhoben:

Un montant est perçu pour une absence ou une annulation. Il est de:

- Fr. 50.- pro Person ab 14 Tage vor Seminarbeginn / par personne dans les 15 jours avant;

- Fr. 100.- pro Person ab 7 Tage vor Seminarbeginn oder Fernbleiben / par personne dans les 7 jours avant le début du séminaire. 